\title{
Some Symmetry Results for the A-Laplacian Equation via the Moving Planes Method*
}

\author{
Zhongbo Fang, Anna Wang \\ School of Mathematical Sciences, Ocean University of China, Qingdao, China \\ Email: fangzb7777@hotmail.com
}

Received July 3, 2012; revised September 1, 2012; accepted September 8, 2012

\begin{abstract}
In this paper, we are concerned with a positive solution of the non-homogeneous A-Laplacian equation in an open bounded connected domain. We use moving planes method to prove that the domain is a ball and the solution is radially symmetric.
\end{abstract}

Keywords: Symmetry; A-Laplacian; Moving Planes Method; Overdetermined Boundary Value Problem

\section{Introduction}

In this paper, we are going to study the symmetry results for the overdetermined problem

$$
\begin{gathered}
\operatorname{div}(A(|\nabla u|) \nabla u)+f(u,|\nabla u|)=0, \text { in } \Omega \backslash\{P\} . \\
u=0, \text { on } \partial \Omega . \\
\frac{\partial u}{\partial v}=c, \text { on } \partial \Omega .
\end{gathered}
$$

Here $\Omega$ is a bounded connected open subset of $R^{n}$ with $C^{2}$ boundary and $P$ is a point in $\Omega$. The function $A:(0, \infty) \rightarrow[0, \infty)$ satisfies the regularity requirement

$$
A \in C^{2}(0, \infty)
$$

and the (possibly degenerate) elliptic condition

$$
\lim _{t \rightarrow 0^{+}} t A(t)=0,(t A(t))^{\prime}>0 \text { for } t>0
$$

$f$ is a continuously differentiable function. $c$ is a constant and $v$ denotes the inner normal to $\partial \Omega$.

J. Serrin proved the radial symmetry for positive solutions of the equation $\Delta u=-1$ in $\Omega$ with the same overdetermined boundary conditions as the above problem, see [1]. N. Garofalo and J. Lewis extended Serrin's result to a larger class of elliptic equations possibly degenerate, including the following p-Laplacian equation $\operatorname{div}\left(|\nabla u|^{p-2} \nabla u\right)=-1$ with the same boundary conditions, see [2]. For the overdetermined elliptic boundary value

\footnotetext{
${ }^{*}$ This work is supported by the Natural Science Foundation of Shandong Province of China (ZR2012AM018).
}

problem $\operatorname{div}(A(|\nabla u|))=-1$ in $\Omega$ with the same overdetermined boundary conditions as above, I. Fragala, I. F. Gazzaola and B. Kawohl used the geometric approach which relies on a maximum principle for a suitable $\mathrm{P}$ function, combined with some geometric arguments involving the mean curvature of $\partial \Omega$ to prove that if the above problem admits a solution in a suitable weak sense, then $\Omega$ is a ball, see [3]. A. Farina and B. Kawohl obtained the same result under removing the strong ellipticity assumption in [4] and a growth assumption in [2] on the diffusion coefficient $A$, as well as a starshapedness assumption on $\Omega$ in [3], see [5]. A. Firenze considered the positive solution of problem (1.1)-(1.3) when it is a p-Laplacian equation in an open bounded connected subset $\Omega$ of $R^{n}$ with $C^{2}$ boundary, see [6]. All of the above motivated us to extend the symmetry result to the non-homogeneous A-Laplacian equation.

Our main result is that for the problem (1.1)-(1.3), if $u$ has only one critical point in $\Omega$, then $\Omega$ is a ball and $u$ is radially symmetric.

Section 2 of this paper is devoted to the main result and a more general version of this theorem. In Section 3, we will present the proof of the main theorem.

Some components, such as multi-leveled equations, graphics, and tables are not prescribed, although the various table text styles are provided. The formatter will need to create these components, incorporating the applicable criteria that follow.

\section{Preliminaries and Statement of Results}

In this section we give some lemma that we shall use and present our main result. 
Lemma 2.1. (The boundary lemma at corner) (Lemma 2 in [1]) Let $\Omega$ be a domain with $C^{2}$ boundary and $T$ be a hyperplane containing the normal to $\partial \Omega$ at some point $Q$. Let $\Omega^{*}$ denote the portion of $\Omega$ lying on some particular side of $T$.

Suppose that $W$ is of class $C^{2}$ in the closure of $\Omega^{*}$ and satisfies the elliptic inequality

$$
L w=\sum_{i, j=1}^{n} a_{i j}(x) w_{i j}+\sum_{i=1}^{n} b_{i}(x) w_{i} \leq 0, \quad x \in \Omega^{*}
$$

where the coefficients are uniformly bounded. We assume that the matrix $a_{i j}$ is uniformly definite

$$
\sum_{i, j=1}^{n} a_{i j}(x) \xi_{i} \xi_{j} \geq k|\xi|^{2}, \quad k=\text { const }>0,
$$

and that

$$
\sum_{i, j=1}^{n}\left|a_{i j} \xi_{i} \eta_{j}\right| \leq K(|\xi \cdot \eta|+|\xi| \cdot|\mathrm{d}|), \quad K=\text { const }>0,
$$

where $\xi=\left(\xi_{1}, \cdots, \xi_{n}\right)$ is an arbitrary real vector, $\eta=\left(\eta_{1}, \cdots, \eta_{n}\right)$ is the unit normal to the plane $T$, and $\mathrm{d}$ is the distance from $T$. Suppose also $w \geq 0$ in $\Omega^{*}$ and $w=0$ at $Q$. Let $S$ be any direction at $Q$ which enters $\Omega^{*}$ nontangentially. Then

$$
\frac{\partial w}{\partial s}>0 \text { or } \frac{\partial^{2} w}{\partial s^{2}}>0 \text { at } Q,
$$

unless $w \equiv 0$.

Our main results are as follows:

Theorem 2.2. Let $\Omega$ be a bounded connected open subset of $R^{n}$ with $C^{2}$ boundary and let $P$ be a point in $\Omega$. Let $u \in C^{2}(\bar{\Omega} \backslash\{P\}) \cap C^{1+\alpha}(\bar{\Omega}), \quad 0<\alpha<1$, be a strictly positive solution of the following overdetermined boundary value problem

$$
\begin{gathered}
\operatorname{div}(A(|\nabla u|) \nabla u)+f(u,|\nabla u|)=0, \text { in } \Omega \backslash\{P\} . \\
u=0, \text { on } \partial \Omega . \\
\frac{\partial u}{\partial v}=c, \text { on } \partial \Omega .
\end{gathered}
$$

Here $f$ is a continuously differentiable function, $A \in C^{2}(0, \infty)$ and

$$
\lim _{t \rightarrow 0^{+}} t A(t)=0,(t A(t))^{\prime}>0 \text { for } t>0 .
$$

$c$ is a constant and $v$ denotes the inner normal to $\partial \Omega$. Assume

$$
|\nabla u|>0 \text { in } \Omega \backslash\{P\},
$$

then $\Omega$ is a ball and $u$ is radially symmetric.

The following remark is a general version of the theorem. It can be viewed as an extension result of p-Laplacian too. As the proof is similar to Theorem 2.2, we omit it.

Remark 2.3. Let $\Omega$ be as in Theorem 2.2 and $D$ be a subset of $\Omega$. Let $u \in C^{2}(\bar{\Omega} \backslash D) \cap C^{1+\alpha}(\bar{\Omega})$ be a strictly positive solution of Equation (2.1) in $\Omega \backslash D$ and verify the boundary conditions; Assume that $D$ is the critical set of $u$, then if $\hat{D}$ denotes the convex hull of $D$,

1) the normal line to $\partial \Omega$ at an arbitrary point of $\partial \Omega$ intersects $\hat{D}$;

2) if $\pi$ is a support plane to $\hat{D}$ through $A \in \partial \hat{D} \cap \Omega$ and $\gamma$ is a ray from $A$ orthogonal to $\pi$ which lies in the half-space determined by $\pi$ not containing $\hat{D}$, then $\gamma$ intersects $\partial \Omega$ exactly in one point.

In what follows we assume that the origin $O$ of the coordinates system is an interior point of $\Omega$, and we denote with $B_{\varepsilon}$ the closure of the ball centered in $O$ with radius $\varepsilon$.

Theorem 2.4. Assume that the hypotheses of Theorem 2.2 hold and furthermore assume that

$$
D \subset B_{\varepsilon} \subset \Omega
$$

for some positive $\varepsilon$. Then

1) $\Omega$ is starshaped with respect to $O$;

2) if

$$
\begin{aligned}
& d=\inf \{\|P-O\|: P \in \partial \Omega\} ; \\
& l=\sup \{\|P-O\|: P \in \partial \Omega\} ;
\end{aligned}
$$

then

$$
l-d \leq \frac{\pi d \varepsilon}{\sqrt{d^{2}-\varepsilon^{2}}} .
$$

\section{Proof of Theorem 2.1}

The technique we are going to use is the moving planes method. For the detailed description about moving planes method, see [1].

Proof. Step 1: To prove $\Omega$ is a ball.

If we can demonstrate that for any point $Q$ on $\partial \Omega, P$ lies on the normal line to $\partial \Omega$ at $Q$, then $\Omega$ is a ball with centre $P$. To do this, we argue by contradiction.

Assume that there exists a point $Q \in \partial \Omega$ such that the normal line $r$ to $\partial \Omega$ at $Q$ does not contain $P$. We choose a coordinate system in $R^{n}$ such that $P \equiv(-\theta, 0, \cdots, 0), \theta>0$, and the $x_{n}$ axis coincides with $r$.

When we use the moving planes method, we choose a family of hyperplanes normal to the $x_{1}$ axis. Define hyperplan $T(\lambda) \equiv\left\{x_{1}=\lambda\right\}$ for any positive $\lambda$; Let $\lambda_{0}$ be the infimum of $\lambda^{\prime}$ s such that $T(\lambda) \cap \bar{\Omega}=\phi$; Define $\Sigma(\lambda)=\Omega \bigcap\left\{x_{1}>\lambda\right\}$ for $\lambda<\lambda_{0}$ and we denote by $\Sigma^{\prime}(\lambda)$ the reflection $\Sigma(\lambda)$ in $T(\lambda)$. Since $\partial \Omega$ is $C^{2}$, for some $\lambda$ close to $\lambda_{0}, v$, we have

$$
\Sigma^{\prime}(\lambda) \subset \Omega \text {. }
$$

As $\lambda$ decreases, condition (3.1) holds until one of 
the following facts happens:

1) $\partial \Sigma^{\prime}(\lambda)$ is internally tangent to $\partial \Omega$ at some point of $\partial \Sigma^{\prime}(\lambda) \backslash T(\lambda)$;

2) $T(\lambda)$ intersects $\partial \Omega$ at some point of $\partial \Omega$.

Let $\lambda^{\prime}$ be the greatest value of $\lambda, \lambda<\lambda_{0}$, such that either condition a) or b) is true. Since $T_{0}$ is orthogonal to $\partial \Omega$ at $Q$, we have $\lambda^{\prime} \geq 0$ and then $P \notin T(\lambda)$ for any $\lambda$ in $\left[\lambda^{\prime}, \lambda_{0}\right]$. This is the crucial point of our proof. We have found a direction such that as the moving plane $T(\lambda)$ moves from $T\left(\lambda_{0}\right)$ to the critical position $T\left(\lambda^{\prime}\right)$, it never intersects $P$, so that the moving planes method may be applied.

Let $x^{\lambda}$ be the reflected point of $x$ in $T(\lambda)$. We defined

$$
\begin{gathered}
v(x)=u\left(x^{\lambda}\right) \text { for } x \in \Sigma(\lambda), \lambda \in\left[\lambda^{\prime}, \lambda_{0}\right], \\
w(x)=v(x)-u(x) .
\end{gathered}
$$

From Equation (2.1) we have for $\lambda^{\prime} \leq \lambda \leq \lambda_{0}$,

$$
\begin{aligned}
& A(|\nabla u|) \Delta u+\frac{A^{\prime}(|\nabla u|)}{|\nabla u|} \sum_{i, j=1}^{n} u_{i} u_{j} u_{i j}+f(u,|\nabla u|) \\
& =0 \text { in } \Sigma(\lambda) .
\end{aligned}
$$

By the definition of $v$, we obtain

$$
\begin{aligned}
& A(|\nabla v|) \Delta v+\frac{A^{\prime}(|\nabla v|)}{|\nabla v|} \sum_{i, j=1}^{n} v_{i} v_{j} v_{i j}+f(v,|\nabla v|) \\
& =0 \text { in } \Sigma(\lambda) \backslash\left\{P^{\lambda}\right\} .
\end{aligned}
$$

Differencing Equations (3.2) and (3.3) yields

$$
\begin{aligned}
& A(|\nabla v|) \Delta v+\frac{A^{\prime}(|\nabla v|)}{|\nabla v|} \sum_{i, j=1}^{n} v_{i} v_{j} v_{i j}+f(v,|\nabla v|) \\
& -A(|\nabla u|) \Delta u-\frac{A^{\prime}(|\nabla u|)}{|\nabla u|} \sum_{i, j=1}^{n} u_{i} u_{j} u_{i j}-f(u,|\nabla u|)=0 .
\end{aligned}
$$

Meanwhile, (3.4) can also be rewritten into

$$
\begin{aligned}
& \{A(|\nabla v|)+A(|\nabla u|)\} \Delta(v-u) \\
& +\sum_{i, j=1}^{n}\left(\frac{A^{\prime}(|\nabla v|)}{|\nabla v|}+\frac{A^{\prime}(|\nabla u|)}{|\nabla u|}\right)(v-u)_{i j} \\
& +\{A(|\nabla v|)-A(|\nabla u|)\} \Delta(v+u) \\
& +\sum_{i, j=1}^{n}\left(\frac{A^{\prime}(|\nabla v|)}{|\nabla v|}-\frac{A^{\prime}(|\nabla u|)}{|\nabla u|}\right)(v+u)_{i j} \\
& =2\{f(u,|\nabla u|)-f(v,|\nabla v|)\} .
\end{aligned}
$$

Denote $f[u] \equiv f(u,|\nabla u|), \quad A[u] \equiv A(|\nabla u|)$,

$$
A^{\prime}[u] \equiv \frac{A^{\prime}(|\nabla u|)}{|\nabla u|} .
$$

Let

$$
a_{i j}(x)=\{A[v]+A[u]\} \delta_{i j}+\left\{A^{\prime}[v] v_{i} v_{j}+A^{\prime}[u] u_{i} u_{j}\right\}
$$

By the mean value theorem, it follows from (3.5) that

$$
\sum_{i, j=1}^{n} a_{i j} w_{i j}+\sum_{k=1}^{n} b_{k} w_{k}+c w=0
$$

where $a_{i j}, b_{k}$ and $c$ are certain functions depending on $u$ and $f$. Here the matrix $a_{i j}$ is uniformly positive definite, since both expressions $A[u] \delta_{i j}+A^{\prime}[u] u_{i} u_{j}$ and $A[v] \delta_{i j}+A^{\prime}[v] v_{i} v_{j}$ have this property (recall that Equation (2.1) is elliptic). So (3.6) is uniformly elliptic with bounded coefficients far from $P^{\lambda}$, i.e. in $\Sigma(\lambda) \backslash B_{\varepsilon}^{\lambda}$ where $B_{\varepsilon}^{\lambda}$ is a ball centered in with radius $\varepsilon$, for any positive $\varepsilon$.

From the boundary condition (2.3) on the normal derivative of $u$, it follows that

$$
w(x, \lambda)>0 \text { in } \Sigma(\lambda)
$$

for some $\lambda<\lambda_{0}$ sufficiently close to $\lambda_{0}$. Let $\lambda^{*}=\inf \left\{\lambda \in\left[\lambda^{\prime}, \lambda_{0}\right]:(3.7)\right.$ holds $\}$. We prove $\lambda^{*}=\lambda^{\prime}$. Assume $\lambda^{*}=\lambda^{\prime}$, by continuity, $w\left(x, \lambda^{*}\right) \geq 0$ in $\Sigma\left(\lambda^{*}\right)$. On the other hand, since $\Omega$ is not symmetric with respect to $T\left(\lambda^{*}\right), \quad w \neq 0$ in $\Sigma\left(\lambda^{*}\right)$. By the strong version of the maximum principle, we obtain $w>0$ in $\Sigma\left(\lambda^{*}\right) \backslash B_{\varepsilon}^{\lambda^{*}}$. Next we observe that $P^{\lambda^{*}}$ can not be a critical point for $w$ since $\nabla v\left(P^{\lambda^{*}}\right)=0$ while

$\nabla u\left(P^{\lambda^{*}}\right) \neq 0$. So as $\varepsilon$ is arbitrarily small, it is $w>0$ in $\Sigma\left(\lambda^{*}\right)$. Since $P^{\lambda^{*}} \notin T\left(\lambda^{*}\right)$, we may apply the Hopf lemma to $w$ at each point of $\Omega \cap T\left(\lambda^{*}\right)$, we get

$$
\frac{\partial w}{\partial x_{1}}>0 \text { on } T\left(\lambda^{*}\right) \cap \Omega
$$

The plane $T\left(\lambda^{*}\right)$ is not normal to $\partial \Omega$ at any point, then from inequality (3.8) and the boundary condition (2.3) on the normal derivative of $u$, we get

$$
\frac{\partial u}{\partial x_{1}}<0
$$

By the definition of $\lambda^{*}$, there exists a sequence $x_{n}$ such that $x_{n} \in \Sigma\left(\lambda^{*}-\frac{1}{n}\right)$ and

$$
w\left(x_{n}, \lambda^{*}-\frac{1}{n}\right) \leq 0
$$

Let $\bar{x}$ be a limit point for $x_{n}$ in the closure of $\Sigma\left(\lambda^{*}\right)$, by continuity $w\left(\bar{x}, \lambda^{*}\right)=0$, thus $\bar{x} \in \partial \Sigma\left(\lambda^{*}\right) \cap T\left(\lambda^{*}\right)$. But from inequality (3.10) and the mean value theorem we get $\frac{\partial u}{\partial x_{1}}(\bar{x}) \geq 0$ and this contradicts condition (3.9). 
So $\lambda^{*}=\lambda^{\prime}$ is proved.

Now we will prove that $u$ must be symmetric with respect to $T\left(\lambda^{\prime}\right)$. Assume $w \neq 0$ in $\Sigma\left(\lambda^{\prime}\right)$, so as we did for $\lambda=\lambda^{*}$, we infer $w>0$ in $\Sigma\left(\lambda^{\prime}\right)$.

Assume next that condition a) holds, then $\partial \Sigma^{\prime}\left(\lambda^{\prime}\right)$ is internally tangent to $\partial \Omega$ at some point $M^{\lambda^{\prime}}$, where $M \in\left\{\partial \Sigma\left(\lambda^{\prime}\right) \backslash T\left(\lambda^{\prime}\right)\right\} \cap \partial \Omega$. Since $P$ is an interior point of $\Omega, P \neq M^{\lambda^{\prime}}$, so that we can apply the Hopf lemma to $w$ at $M$ and we obtain

$$
\frac{\partial w}{\partial v}\left(M, \lambda^{\prime}\right)>0
$$

where $v$ is the inner normal to $\partial \Omega$ at $M$. For

$$
\frac{\partial w}{\partial v}\left(M, \lambda^{\prime}\right)=\frac{\partial u}{\partial v}\left(M^{\lambda^{\prime}}\right)-\frac{\partial u}{\partial v}(M)=c-c=0,
$$

we get the contradiction. Hence condition 2) must be true, i.e. $T\left(\lambda^{\prime}\right)$ is orthogonal to $\partial \Omega$ at some point $B$. From the boundary condition (2.3) and the definition of $w$ it follows that all the first and second derivatives of $w$ vanish at $B$. On the other hand, as $P^{\lambda^{\prime}} \neq B$, Equation (3.6) is uniformly elliptic with bounded coefficents in a neighborhood of $B$, so that the boundary lemma at corner in [1] lemma 2, may be applied to $w$. Let $s$ be a direction which enters $\partial \Sigma\left(\lambda^{\prime}\right)$ nontangentially at $B$, then by the Serrin's lemma

$$
\frac{\partial w}{\partial s}\left(B, \lambda^{\prime}\right)>0 \text { or } \frac{\partial^{2} w}{\partial s^{2}}>0
$$

Then we have again a contradiction with the derivatives of $w$ at $B$, so $w \equiv 0$ in $\Sigma\left(\lambda^{\prime}\right)$. But this last ine- quality can not be true since otherwise $w$ would be a function symmetric in $T\left(\lambda^{\prime}\right)$ whose only critical point is not on $T\left(\lambda^{\prime}\right)$.

This completes the proof of Theorem 2.1.

\section{REFERENCES}

[1] J. Serrin, "A Symmetry Problem in Potential Theory," Archive for Rational Mechanics and Analysis, Vol. 43, No. 4, 1971, pp. 304-318. doi:10.1007/BF00250468

[2] N. Garofalo and J. Lewis, "A Symmetry Result Related to Some Overdetermined Boundary Value Problems," American Journal of Mathematics, Vol. 111, No. 1, 1989, pp. 9-33. doi: $10.2307 / 2374477$

[3] I. Fragala, I. F. Gazzaola and B. Kawohl, "Overdetemined Boundary Value Problems with Possibly Degenerate Ellipticity: A Geometry Approach," Mathematische Zeitschrift, Vol. 254, No. 1, 2006, pp. 117-132. doi:10.1007/s00209-006-0937-7

[4] G. A. Philippin, "Application of the Maximum Principle to a Variety of Problems Involving Elliptic Differential Equations," In: P. W. Schaefer, Ed., Maximum Principles and Eigenvalue Problems in Partial Differential Equations, Pitman Research Notes in Mathematics Series, Longman SciTech., Harlow, 1988, pp. 34-48.

[5] A. Farina and B. Kawohl, "Remarks on an Overdetermined Boundary Value Problem," Calculus of Variations and Partial Differential Equations, Vol. 31, No. 3, 2008, pp. 351-357.

[6] A. Firenze, "A Symmetry Result for the p-Laplacian Equation via the Moving Planes Method," Applocable. Analysis, Vol. 55, No. 3-4, 1994, pp. 207-213. 